\title{
Web Applications Design with a Multi-process Approach
}

\author{
Semia Sonia Selmi, Naoufel Kraiem, and Henda Ben Ghezala \\ ENSI, National School of Computer Science, 2010 Manouba, Tunisia \\ Semiasonia.selmi@riadi.rnu.tn, Naoufel.kraiem@ensi.rnu.tn \\ Henda.BG@cck.rnu.tn
}

\begin{abstract}
This paper deals with a new approach WApDM (Web Applications Design Method) for Web Applications Design. The WApDM is a multi-process approach whish covers all aspects should be considered during design of a Web application. The proposed approach is specified with the MAP formalism. The use of the MAP is three fold: (a) the MAP process meta-model is adapted to the specification of complex methods, (b) MAP introduces more flexibility in the method and (c) implicit information of the method are made explicit during the specification activity.
\end{abstract}

\section{Introduction}

Considerable attention has been given to Web engineering: the discipline which is concerned with the establishment and the use of engineering and management principles and disciplined and systematic approaches to the successful development, deployment and maintenance of high quality web-based applications.

In order to manage the overall complexity of development, several methods and models have been proposed. They should provide guidelines for performing activities and suitable models for expressing the results. Our reflection on this issue, which is shared by [8] [6] and others authors, is that the development of a web application should not be an event, but a process. This process will consist of a set of manageable activities. As stressed by [8], it is important to be guided by a sequence of steps to be performed, to know how the different steps co-operate and how they fit into the development process as a whole. Each design activity should address different concerns at the proper stage and at the proper level of abstraction.

However, we have concluded that there is a number of gaps in existing modeling methods, particular with regard to the level of guidance and flexibility provided. In fact, major existing methods do not consider systematically all features should be implied during design of a complex web application such as a Web Information System (WIS) such users profiles or offered services. We argue that the design of a complex web application should comprise the following activities that can not be neglected:

- organization and presentation of information which form the navigation support

- modeling of functional aspect dealing with services modeling offered by application

- considering users and/or adaptation modeling in the purpose to give impression that application is specially developed for user.

In spite of the importance of each of the aforementioned activities, the focus of existing methods is still different, some try to address many aspects in the design process, others try to detail in depth one or two of them. 
Other important issue characterizing existing methods is that they are prescriptive. Their processes specify only what must be done; they are already predefined. However, a design process does not always specify what must be done but contains some specification of what can be done. Therefore, it contains a number of alternative ways of doing a task and a selection of the particular alternative should be done dynamically, depending upon the situation in which designer is placed.

In this context, we consider design process as a decision making one. In fact, at any time a designer is in a situation that he views with some specific intention. His reaction depends on both these factors; i.e. on the context in which he has been involved. Methods should provide designer with the ability to decide how he can proceed according to his evaluation of the situation. Designing a WIS, for instance, requires a number of decisions to be made: what to consider as features?, what technique shall use?, the most appropriate solution?, etc.

In this research, we propose the WApDM method, a meta-approach that covers existing methodologies transparently. It takes as input the application requirements and decides which process to follow. It provides designer with the ability to move through the basic design steps. The work is partially motivated by conclusions derived in both Method Engineering discipline and Web Engineering discipline [4] [10]. It was observed that there is no existing full-featured approach that one can use to develop different kinds of applications with different requirements. Consequently, if one wants to develop more than one application, he might need to use more than one methodology.

Considering the design process as a process of decision taking, the proposed method provides more flexibility since it allows designer to decide in each design process step, and thus, personalize his process.

With regards to purposes to reach, we have opted to adopt the meta-model MAP as modeling formalism, offering different ways to guide achievement of design activities. The MAP provides guidance to a lower level abstraction through associated guidelines.

The remainder of this paper is organized as follows. We provide in the second section an overview of WApDM method, its principle and its components. In the third section, we present the MAP process meta-model used as modeling formalism in section 4. Finally, we summarize our conclusions and future works.

\section{Overview of WApDM Method}

The WApDM is a multi-process method: it offers panoply of different processes for the design of web applications such as WIS, adaptive applications, e-commerce applications, etc. It supports the most part of design process. Each of activities addresses a particular concern and is accomplished separately.

The WApDM is a meta-approach that covers existing methods transparently. Typically methods consider the design process in terms of process phases and their deliverables, often models.

It is important to note, at this level, that design models should answer the need of formalizing the design of hypermedia applications. They should also help to reason in a structured way on the aspects that are specific to hypermedia design. 
A typical web design method has the following phases [5]:

- Conceptual design: describes the organization of the information managed by the application, in terms of pieces of content that constitute its information base and their semantic relationships. Modelling aims to construct a conceptual model without commitment to any specific detail for navigation paths, presentation and interaction aspects.

- Navigation design: concerns the facilities for accessing information and for moving across the application content. The navigation structure should be carefully designed through a navigation model by providing the user with the comfortable navigation spaces.

- Presentation design: affects the way in which the application content and the navigation commands are presented to the user. This is described in a presentation model.

- Besides, and due to the evolution of the web and therefore of web applications, others phases are recognized namely:

- Requirements analysis: gathering and forming the specification of users and/or stakeholders requirements. This step delivers a requirements analysis model.

- Adaptation modelling: the success of web applications is largely dependent on user satisfaction which is achieved by, for example, easy-to-use interface and well structured navigational architectures. The most effective technique to leverage these features is adaptation. It consists on delivering them to the right user at the right time in the right format. This phase presents the objects that participate in the adaptive functionality and describes how this adaptation is performed [9]. It aims to construct an adaptation model which is based on a user model.

- User modelling: aims to construct a user model which contains information that represents the view the system has of the knowledge, goals and/or individual features of user.

- Business process modelling: apart from simple web sites, web applications are derived from conventional transaction processing systems. These applications support critical business processes and workflows that are important part of the organisation's core business model. These business functions must be supported and consequently web design methods need to provide the ability to represent these functions and their related design artefacts. It is an important activity in particular for the e-business applications design. It helps designers and developers, for instance, in identifying and understanding the relevant elements in a specific domain and their relationships [15].

The first four phases could be supported during the design process of any web application. However, others are specific to some web applications types.

In fact, adaptation modelling and user modelling phases are both performed when designing adaptive applications as it is defined in [2].

User modelling phase can be also performed in isolation when designing a usermodel based application. Designer, in such case, intends to adopt a user-centred approach starting design process with user requirements and characteristics. This has the advantage to solve disorientation and cognitive overload problems.

As for Business process modelling, is particularly recognised for e-commerce applications. As it is referenced by [15] and many others researchers, it helps in 
identifying and understanding the relevant elements in a specific domain and their relationships. Most existing web design methods fail to address the modelling of functionality in web applications. Their focus has been on the organisation, presentation and navigation aspects while business functions have been consistently overlooked.

Our reflection in this point, that the business process modelling phase should be performed during any web application design. Designer can judge about the domain complexity in term of service complexity. For instance, if services of current domain are of a high complexity, designer should construct the business process model in order to better understand domain. Otherwise, such case of simple web site of very low information complexity, it is useless to perform this step.

As we can notice, design phases mentioned above can be classified according to dependency of their deliverable models on current web application to be designed. In fact, all aspects of organising structure, choosing content or presentation modalities are aspects that totally depend on application. In contrast, others aspects as user modelling or adaptation modelling are considered at a high level of modelling and do not depend directly on application. Besides, the first three activities (structure, navigation and presentation) are typically delivered during web design [5].

Consequently, we have distinguished two models classes: Application models class and Business models class. The first class comprises application domain model, navigation model and presentation model. The second class is containing requirements analysis model, adaptation model, user model and business process model.

The WApDM method covers all aforementioned design phases and adopt the process meta-model MAP as modeling formalism. In the following section, we present an overview of the MAP and its associated guidelines. In the section IV, the formalization of the WApDM method with MAP is detailed.

\section{Overview of MAP}

A MAP is a process model which allows designing several processes under a single representation. It is a labelled directed graph with intentions as nodes and strategies as edges between intentions [1]. The directed nature of the graph shows which intentions can follow which ones.

According to the meta-model illustrated in Fig. 1, a Map is composed of one or more sections. A section is a triplet <source intention I, target intention J, strategy $\mathrm{Sij}>$ that captures the specific manner to achieve the intention $\mathrm{J}$ starting from the intention I with the strategy Sij. An intention is expressed in natural language and is composed of a verb followed by parameters. Each Map has two special intentions "Start" and "Stop" to begin and end the navigation in the Map. Each intention can only appear once in a given Map.

1. A guideline named "Intention Achievement Guideline" (IAG) is associated to each section providing an operational mean to satisfy the target intention of the section.

2. "Strategy Selection Guideline" (SSG) determines which strategies connect two intentions and helps to choose the most appropriate one according to the given situation. It is applied when more than one strategy exists to satisfy a target intention from a source one. 


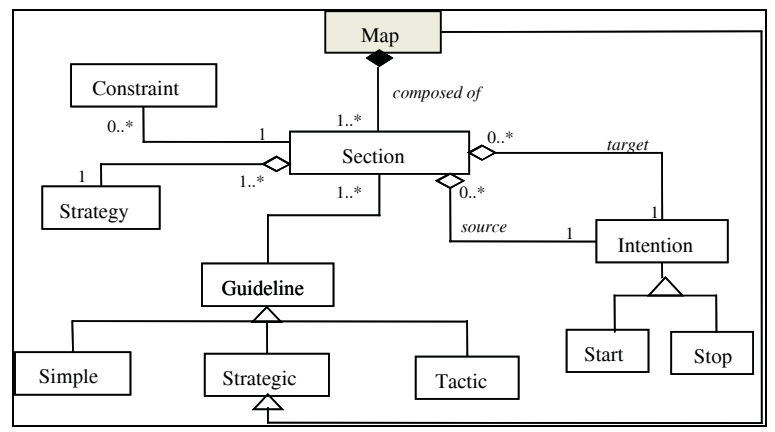

Fig. 1. The MAP process meta-model

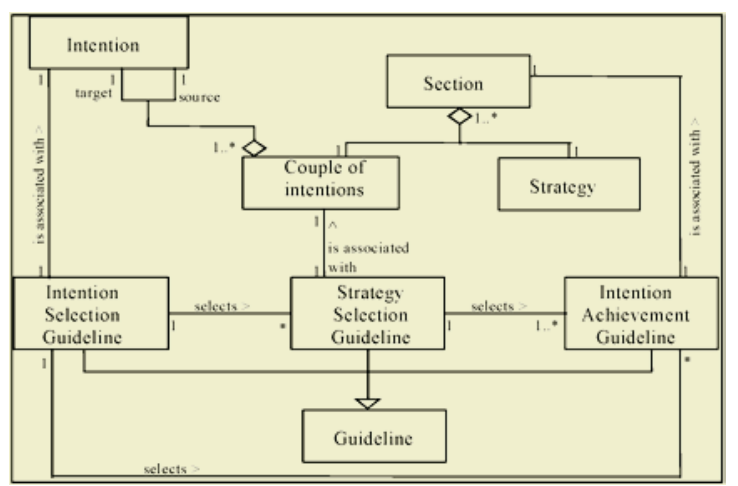

Fig. 2. Guidelines associated with the MAP

3. "Intention Selection Guideline" (ISG) determines which intentions follow a given one and helps in the selection of one of them. It results in the selected intention and the corresponding set of either IAGs or SSGs. The former is valid when there is only one section between the source and target intentions, whereas the latter occurs when there are several sections.

Fig. 2 shows that: (1) for a section $\left\langle\mathrm{I}_{\mathrm{i}}, \mathrm{I}_{\mathrm{j}}, \mathrm{S}_{\mathrm{ij}}\right\rangle$, there is an IAG, (2) for a couple of intentions $<\mathrm{I}_{\mathrm{i}}, \mathrm{I}_{\mathrm{j}}>$, there is an SSG, and (3) for an intention $\mathrm{I}_{\mathrm{i}}$, there is an ISG.

\section{Formalizing WApDM Method with MAP}

Processes identified using the meta-model MAP integrate all main activities of web applications design in different ways that designer can follow in order to achieve design process and, thus, have a web application of quality.

\subsection{Presentation of the Generic MAP of Process Model}

As shown in Fig. 3, the Map contains two core intentions Define application model and Define Business model in addition to Start and Stop intentions. They correspond to the definition of the different models, deliverables of design activities. 


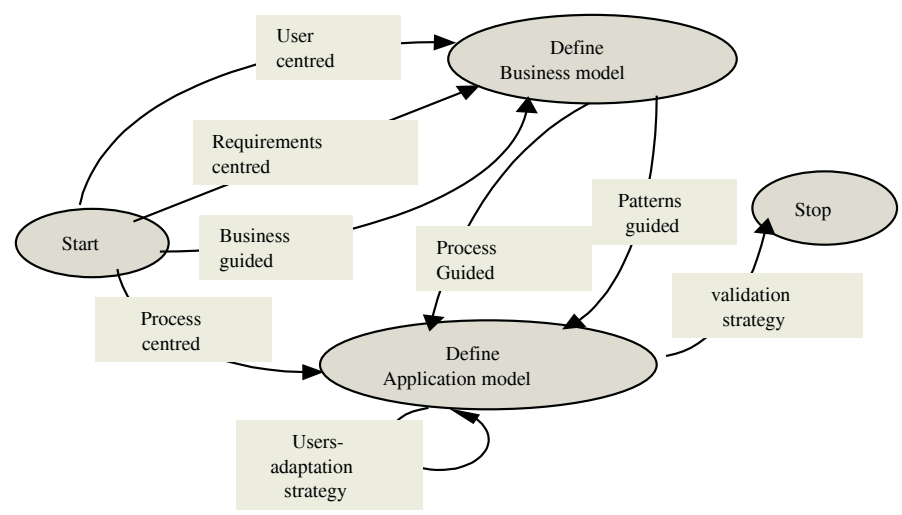

Fig. 3. Generic process model of Web applications design

The generic process model presented in Fig. 3 provides a rich collection of process chunks. These allow identifying several strategies for each of aforementioned intentions.

To achieve the first intention Define Business model, the process model proposes three strategies: the user-centered strategy aims to construct an adaptive application and/or a user-based application. At this level, business model will correspond to user model and/or adaptation model; the Requirements-centered strategy supports users and/or stakeholders requirements analysis. Finally, Business-guided strategy corresponds to the definition of a Business process model. This strategy can be applied during design of any web application and in particular complex applications. These three strategies can be performed alternatively or together.

The second intention can be achieved by using either process-guided strategy or patterns-guided strategy. The patterns-guided strategy is applied when the designer decides to use a catalogue of patterns that helps him during the achievement of Define application model intention. The process-guided strategy is applied when designer decides to follow provided guidelines associated to WApDM method.

Process model generation is under the control of guidelines. Table 1 recapitulates all guidelines associated to the MAP of Fig. 3.

To allow designer navigation in map, WApDM provides a set of factors called Situational Factors. These factors help designer to choose the appropriate strategy and appropriate intention among several presented in the MAP. We have identified a number of factors such: the type of the application designer intends to design, complexity of application in term of offered services, similarity of the application with other applications already designed in same domain, degree of user-application adaptation, problem clarification and notation standardization.

In the following section, we present how they can help designer in navigation in map through the choice of the appropriate intention or strategy.

\subsection{Example}

The sequence of intentions shown in Fig. 4 is an example of a path that could be followed to design a web application. A more in-depth analysis of the process shows that designer is guided in very deep and flexible ways. 
Table 1. Guidelines of WApDM MAP

\section{$<$ (Start), Define Business Model using User centred strategy $>$}

$<$ (Start),Define Business Model using Requirements centred strategy $>$

$<$ (Start), Define Business Model using Buisness guided strategy $>$

$<$ (Start), Define Application Model using Modelling centred strategy $>$

$<$ (Define Business Model), Define Application Model using Process guided strategy $>$

$<$ (Define Business Model), Define Application Model using Patterns guided strategy $>$

$<$ (Define Application Model), Define Application Model using Users adaptation strategy $>$

$<$ (Define Application Model), Stop using Validation strategy $>$

\section{Strategy Selection Guidelines}

$<$ (Start), progress to Define Business Model $>$

$<$ (Define Business Model), progress to Define Application Model $>$

$<$ (Start), Progress from Start $>$

$<$ (Define Application Model), Progress from Define application model $>$

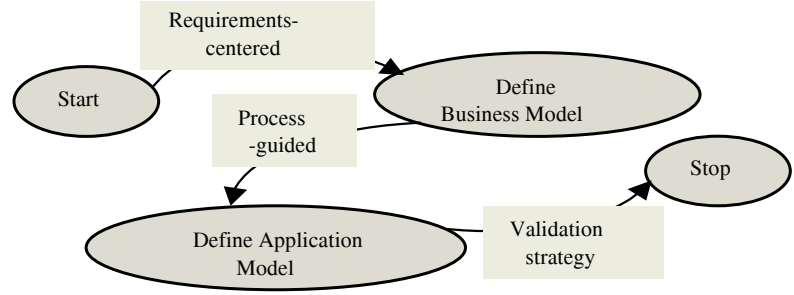

Fig. 4. Example of a web application design path

Sections of WApDM process model are refined to a lower level of abstraction proposing various techniques available to achieve the corresponding intentions.

Guidelines associated to sections included in the path of Fig. 4 namely $<$ Start, Define Business model, Requirements-centred strategy $>$ and $<$ Define Business model, Define Application Model, process-guided strategy $>$ are shown respectively in Fig. 5 and Fig. 6.

Starting from Start intention and by choosing the following section $<$ Start, Define Business model, Requirements-centred strategy $>$, designer is faced to a cluster providing three alternative strategies to achieve Define requirements analysis model intention. As shown in Fig. 5, designer can either use Goal-driven approaches or Scenario-based approaches or else Goal-Scenario based approaches. Each of these strategies is refined to guide designer in defining a requirements analysis model.

Goal-driven approaches model organizational objectives so as to relate them to the functions of the systems. They aim at the conceptualization of purposeful systems only and represent the Why part of system requirements.

The scenario-based approach is an alternative approach to requirements engineering. By focusing on the users' view points, scenario-based approaches help in modeling purposeful system usage from which useful system functions can be derived.

In order to overcome some of the deficiencies and limitations of both approaches used in isolation, designer can combines goal modeling and scenario authoring through a goal/scenario based approach. For instance, designer can adopt CREWSL'Ecritoire method [12]. 


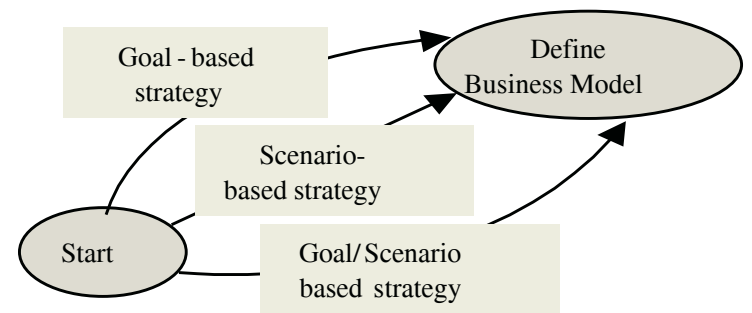

Fig. 5. Cluster associated to <Start, Define Business model, Requirements-centered strategy $>$ section

Once Define Business Model intention is achieved, consisting, during this path, to the definition of a requirements analysis model, designer should realize Define Application Model intention by following the process-guided strategy.

At a lower level of granularity, the following section $<$ Define Business model, Define Application Model, process-guided strategy $>$ is refined with a strategic guideline: MAP as shown in Fig. 6.

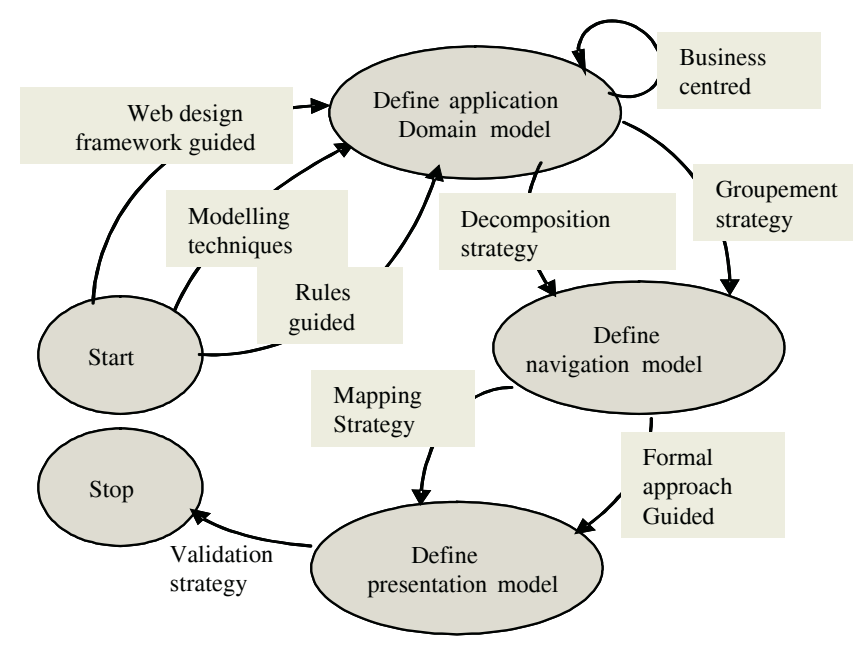

Fig. 6. MAP of <Define Business model, Define Application Model, process-guided strategy> section

The above Map provides panoply of paths and strategies from start and stop intentions. It contains three core intentions Define application domain model, Define navigation model and Define presentation model.

Beginning from the Start intention, designer is faced to three alternatives or manners to achieve Define application domain model intention. This is a Ssg which its body is a hierarchy of contexts. This Ssg is a choice context offering three alternatives.

The Ssg signature $<$ Business model, state (Business model) $=$ defined), progress to Define application domain model $>$ associates the intention of progressing towards the target to define application domain model when the business model has been defined. 
Each of these alternatives proposes the selection of an Intention Achievement Guideline to define Application domain model. The modeling techniques strategy is applied when designer decides to start from scratch and to adopt a well known conceptual data model like ER model [3] or any Object-Oriented technique [13] to define application domain model.

By applying web design framework-guided strategy, designer has experience in current domain and has already designed similar applications in similar domain. The third strategy rules-guided strategy is followed when designer has adopted in previous step the user interaction diagram as mean for the analysis requirements. In this case, he can just apply given rules and construct the corresponding application domain model. Arguments (a1, a2 et a3) are proposed to guide the designer in the selection of the appropriate strategy and associated guideline.

The next step in the process, shown in Fig. 6, is the definition of a navigation model. Once the application domain model is defined, designer can progress to achieve Define navigation model intention either by using Decomposition strategy, consisting on following more than one step or Groupement strategy. The both refer to manner that designer can apply to define navigation model. In general, the navigation model design can be achieved following only one step and it is referenced by Groupement strategy or more than one and it is referenced by Decomposition strategy.

Finally, designer can achieve Define presentation model intention. Fig. 7 shows that designer, at this step of design, is also faced to a SsG composed of two alternatives. He can choose to achieve Define presentation model intention using formal approach-guided strategy and so adopt formal approaches such as ADV Charts. Or, he can proceed by only mapping navigational objects to presentation objects, following, so, the mapping strategy. This strategy can be followed in case of simple web site design of low complexity in term of both information and application complexity.

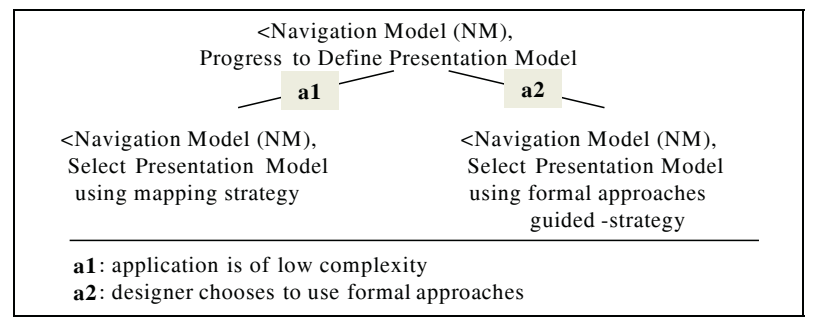

Fig. 7. Details of SSG $<$ (Navigation Model(NM), state(NM=defined), Progress to Define Presentation model>

All these strategies are detailed in a lower level of abstraction in order to provide a more effective guidance to designer. Moreover, designer is guided and directed with respectively guidelines and/or associated arguments which help him to select the best strategy according to its purpose and current situation.

\section{Conclusion}

The paper has presented the WApDM method for the design of web applications. The proposed method is a multi-process approach. It is formalized with the multi-model 
process MAP. Although identifying intentions and specially strategies and organising them was not always an easy task, we observed the following additional important benefits:

- specifying web design methods using the map formalism raised details and levels of abstraction,

- the knowledge underlying the web design is made more explicit,

- web design process map specifies explicitly "why", "how" and "when" to use process chunks. Obviously, these qualitative observations have to be validated with more detailed experiments, e.g. empirical studies.

\section{References}

1. Benjamen, A.: Une approche multi-démarches pour la modélisation des démarches méthodologiques. PhD Thesis, University Paris 1(1999)

2. Brusilovsky, P.: Methods and Techniques of Adaptive Hypermedia. Int. J. User Modeling and User-Adapted Interaction (1996b). Kluwer Academic Publishers Vol 6, 2-3, 87-129

3. Chen, P.P.: The Entity-Relationship Model: toward a unified view of data. ACM TODS 1 (1976) 1, 9-36

4. Christodoulou, S.P., Styliaras, G.D., Papatheodorou, T.S.: Evaluation of hypermedia application development and management systems. Hypertext' 98

5. Fraternali, P.: Tools and Approaches for Developing Data-Intensive web Applications: A Survey. ACM Computing Surveys (1999)

6. Ginige, A.: Web Engineering: Methodologies for Developing Large and Maintainable. Proc IEEE International Conference on Networking the India and the World CNIW-98, Ahmedabad, India

7. Isakowitz, T., Stohr, E.A., Balasubramanian, P.: RMM: a Methodology for Structured Hypermedia Design. ACM Communications (1998) vol. 38, no. 8, 34-44

8. Jacobson, I.: Object-oriented Software Engineering: A Use case driven Approach.Addison Wesley (1992).

9. Koch, N.: Software Engineering for Adaptive Hypermedia Systems-Reference Model. Modelling Techniques and Development Process, Ph.D Thesis (2001), Fakultät der Mathematik und Informatik, Ludwig-Maximilians-Universität München

10. Lee, H., Lee, C., Yoo C.: A Scenario-based Object-Oriented Methodology for Developing Hypermedia Information Systems. Proc of 31st Annual Conference on Systems Science (1998), Sprague R. (Ed.)

11. Rolland, C., Prakash, N.: A proposal for Context-specific Method Engineering. IFIP TC8 Working Conference on Method Engineering (1996), Atlanta, Georgie, USA

12. Rolland, C., Ben Achour, C.: Guiding the Construction of Textual Use Case Specification. In Data \& Knowledge Engineering Journal (1998b), Vol 25, No 1-2, 125-160, (ed. P. Chen, R.P. van de Riet), North Holland, Elsevier Science Publishers

13. Rumbaugh, J., Blaha, M., Premerlani, W., Eddy, F., Loresen, W.: Object-oriented modeling and design. Prentice Hall international (1991)

14. Takahashi, K., Lang, E.: Analysis and Design of Web based Information Systems. Sixth International World Wide Web Conference (1997)

15. Ushold, M., King, M.: Toward a Methodology for Building Ontologies. Workshop on Basic Ontological Issues in Knowledge Sharing (1995), held in conjunction with IJCAI-95, Montreal 